\title{
CRIPTOMOEDAS: FORMAÇÃO DE CARTEIRAS DE INVESTIMENTO ATRAVÉS DO MODELO DE MARKOWITZ
}

\author{
Felipe Fortuna Lucas \\ Universidade Federal do Estado do Rio de Janeiro \\ Avenida Pasteur, 458, CCET, Urca, Rio de Janeiro. \\ felipe.lucas@uniriotec.br \\ Andréa Soares Bonifácio \\ Universidade Federal do Estado do Rio de Janeiro \\ Avenida Pasteur, 458, CCET, Urca, Rio de Janeiro. \\ andreabonifacio@uniriotec.br
}

\section{RESUMO}

O objetivo deste trabalho é aplicar as ferramentas de otimização para o mercado de Criptomoedas. Com a crescimento deste mercado, surge a necessidade de estudar a relação risco-retorno deste tipo de investimento e analisar as oportunidades nele presentes. Serão utilizados dados históricos das Criptomoedas, de Julho de 2017 a Junho de 2018, e através da utilização do modelo de Markowitz (1952) encontrar o portfólio de investimento que ofereça a melhor relação entre risco e retorno.

Palavra-chave: Programação Quadrática; Finanças; Diversificação de Portfólio.

\begin{abstract}
The purpose of this article is to apply the optimization tools to the crypto currency market. With the grown seen in this market, it seems essential to extend the study on the risk return ratio for this type of investment and analyze the opportunities present in this market. Crypto currency historical data are used for the period July/2017 to June/2018, applying the Markowitz Model (1952) to find the investment portfolios that offer the best risk and return ratio.
\end{abstract}

Keywords: Quadratic Programming; Finance; Portfolio Diversification.

\section{Como Citar:}

LUCAS, Felipe Fortuna; BONIFÁCIO, Andréa Soares. Criptomoedas: Formação de Carteiras de Investimento Através do Modelo de Markowitz. In: SIMPÓSIO DE PESQUISA OPERACIONAL E LOGÍSTICA DA MARINHA, 19., 2019, Rio de Janeiro, RJ. Anais [...]. Rio de Janeiro: Centro de Análises de Sistemas Navais, 2019. 


\section{INTRODUÇÃO}

A partir do ano de 2014, período em que o mercado financeiro brasileiro se mostrou bastante instável devido à crise econômica, foi possível notar uma maior aversão ao risco por parte dos clientes e o surgimento de novos modelos de investimentos. Neste contexto surgiu a plataforma de investimentos Bitcoin, que mostrou bons resultados nos primeiros anos de aparecimento no mercado. (ULRICH, 2014)

Através do estudo de carteiras de investimentos, Harry Max Markowitz (1952) desenvolveu a teoria moderna de portfólio a partir da qual foram iniciados os estudos sobre a diversificação de ativos no mercado financeiro, "estes estudos apresentaram pela primeira vez um embasamento teórico matemático em relação à redução de risco resultante da diversificação." (COSTA; ASSUNÇÃO, 2005)

Markowitz (1959) desenvolveu a primeira teoria sobre o comportamento dos ativos de risco e a partir de dados estatísticos e demonstrações matemáticas destacou a importância de compor uma carteira de investimento com ativos de diferentes setores da economia, o que reduz de forma significativa o risco inerente àquele conjunto de ativos, para um dado retorno esperado. (COLIN, 2011).

Neste artigo, seguimos a aplicação do modelo de Markowitz, conforme a realizada em Pereira e Henrique (2016), cuja implementação do modelo foi através de um problema de programação não linear. A Programação não linear é uma ferramenta da Pesquisa Operacional (PO) para encontrar soluções ótimas em problemas que tenham o objetivo de otimizar (através da maximização ou minimização) funções não lineares levando em consideração restrições apresentadas pelo problema (COLIN, 2011).

A elaboração deste trabalho se justifica pelo crescimento dos investimentos em criptomoedas em todo o mundo, gerando diversas discussões e comparações dos critérios de investimento com o mercado de ações. Portanto, será aplicado o modelo de Markowitz, historicamente utilizado no mercado de ações, para o mercado de criptomoedas, que apesar de se mostrar um mercado com uma notável volatilidade, possui características em comum com o mercado de ações.

O objeto de análise compreende as cinco criptomoedas com maior volume de capitalização no período de julho de 2017 a junho de 2018. Aplicou-se o modelo de Markowitz para obter seis carteiras com esses ativos considerando um dado retorno esperado.

No decorrer deste trabalho, são apresentadas as restrições impostas pelo modelo para assim aplicar a Teoria Moderna de Portfolio buscando a carteira de investimento que possua a melhor relação risco-retorno. Na Seção 2 será apresentado o referencial teórico do estudo. $\mathrm{Na}$ Seção 3, aplicamos o modelo de Markowitz numa carteira composta por criptomoedas. Na Seção 4 é realizada a análise dos resultados e na Seção 5 apresentamos as conclusões desse estudo.

\section{REFERENCIAL TEÓRICO}

Nesta etapa, apresenta-se o referencial teórico das análises de otimização em carteiras de investimentos, os conceitos estatísticos estudados por Markowitz (1952), o Índice Sharpe e os conceitos de risco. 


\subsection{OTIMIZAÇÃO EM CARTEIRAS DE INVESTIMENTOS}

Spronk, Steuer e Zopounidis (2005) estudam o rápido aumento da complexidade das decisões financeiras, o que levou ao desenvolvimento e implementação de metodologias que dão suporte à tomada de decisão no campo.

A literatura indica a importância da diversificação de ativos na composição de uma carteira de investimento. Elton et al. (2004) em seu livro sobre Moderna Teoria de Carteiras e Análise de Investimentos, afirma que "os investidores não devem aplicar, e na verdade não aplicam, em um único ativo; eles investem em grupos ou carteiras de ativos."

Costa e Assunção (2005) afirmam que há um conceito empiricamente aceito de que a diversificação de ativos diminui o risco de um investimento.

Diversos trabalhos publicados trazem a aplicação do Modelo de Markowitz para encontrar carteiras de investimentos ótimas. O modelo de Markowitz é considerado o marco inicial sobre os estudos de carteiras de investimento e que introduzem a Teoria Moderna de Investimento (ou Teoria Moderna de Portfolios) (COSTA e ASSUNÇÃO, 2005).

Pereira e Henrique (2016), aplicaram o modelo de Markowitz para montar cinco carteiras com ativos de empresas de diversos setores com ações comercializadas na BM\&FBovespa, de modo a obter a composição mais adequada aos diversos tipos de investidores.

Gonçalves Jr, Montevechi e Pamplona (2002) aplicam a programação linear na seleção de carteiras usando planilhas eletrônicas com o objetivo de auxiliar pequenos investidores na escolha da melhor carteira de investimentos.

Fonseca (2011) investigou a eficiência de diversas carteiras com base na teoria desenvolvida por Markowitz. Os resultados encontrados mostraram o melhor desempenho da carteira obtida através do uso do modelo de Markowitz.

De modo similar ao estudo anterior, Souza et al. (2017) analisaram os 59 ativos que compõem o Ibovespa, no quadrimestre de maio a agosto de 2016. Através de uma comparação entre a carteira otimizada, obtida pela aplicação do modelo de Markowitz e a carteira criada pelo Ibovespa, verificou-se que aquela superou o retorno desta em 107,84\%, obtendo ainda um risco inferior. Num segundo momento, no entanto, o Ibovespa fez-se mais eficiente que a carteira otimizada, de maio a agosto de 2016; porém, o retorno e o risco foram semelhantes àquele. Concluiu-se nesse estudo que a carteira otimizada, quando não consegue ser superior ao Ibovespa, apresenta um retorno próximo devido ao risco.

O estudo de Marques et al. (2013), apresenta uma comparação do modelo de Markowitz com o desempenho da carteira de ações do IBOVESPA, através da utilização do método para encontrar a carteira ótima (com o menor risco e maior retorno) e comparando-a com o retorno dado pelo benchmark no mesmo período estudado pelo modelo.

No estudo de Lima, Guimarães Júnior e Távora Júnior (2010) utilizou-se o modelo da Teoria das Carteiras proposto por Markowitz para a criação de uma carteira de variância mínima para o mercado de ações brasileiro, a partir da análise da série temporal de cotações de ativos da BOVESPA (Bolsa de Valores de São Paulo), por meio de planilhas eletrônicas. Como metodologia para o desenvolvimento dessas carteiras foi utilizado a ferramenta Solver do MS Excel para desenvolver um problema de programação linear.

\subsection{CRIPTOMOEDAS}

Segundo ULRICH (2014) o conceito de criptomoedas surgiu após a crise financeira no ano de 2008, um mês após a quebra do banco de investimentos Lehman Brothers, com o lançamento da moeda digital Bitcoin, por Satoshi Nakamoto. 
ULRICH (2014) cita ainda que os motivos fundamentais que impulsionaram a criação do Bitcoin são, portanto, evidentes: um sistema financeiro instável e com elevado nível de intervenção estatal e a crescente perda de privacidade financeira.

"O Bitcoin é uma forma de dinheiro, assim como o real, o dólar ou o euro, com a diferença de ser puramente digital e não ser emitido por nenhum governo. O seu valor é determinado livremente pelos indivíduos no mercado. Para transações online, é a forma ideal de pagamento, pois é rápido, barato e seguro.” (ULRICH, 2014, pg. 15)

$\mathrm{O}$ autor mostra que a alta volatilidade do Bitcoin testemunhada em alguns períodos específicos ao longo dos anos de 2011 e 2013 complica a análise dos investidores, porém o autor destaca que o aumento de investidores tende a estabilizar o preço desses ativos no decorrer do tempo.

O que permitiu que a criptomoeda fosse viabilizada foi a criação do blockchain, que segundo Crosby (2016) se trata de um banco de dados de registros, onde todos os eventos ocorridos são armazenados, garantindo a autenticidade dos dados inseridos e a grande dificuldade de alteração dos dados devido ao encadeamento de blocos.

Com o sucesso do mecanismo do blockchain na Bitcoin, outras moedas foram surgindo com o tempo, reduzindo a dominação da Bitcoin no mercado de criptomoedas.

"O Bitcoin era detentor de aproximadamente 91,3\% do mercado, seguido por baixa participação de outras criptomoedas, até o surgimento do Ethereum em 2015. Desde então, o quadro mudou: a fatia do mercado relacionada ao Bitcoin foi reduzida para $39,8 \%$, enquanto a do Ether (criptomoeda referente ao Ethereum) subiu para 28,5\%." (MASCARENHAS et al, 2018)

Hoje, segundo a plataforma Coinlib, o mercado conta com mais de cinco mil diferentes tipos de criptomoedas, portanto, mesmo com o crescimento deste mercado o Bitcoin continua com a maior fatia, possuindo um volume da capitalização de aproximadamente 115 bilhões de dólares, enquanto a segunda colocada Ethereum, possui capitalização de 27 bilhões de dólares.

\subsection{DIVERSIFICAÇÃo DE RISCO}

O risco de uma carteira de investimentos pode ser analisado através da variação de preço em relação ao retorno esperado do ativo. (ASSAF NETO, 2014)

Segundo Jorion (1997, pg. 61) "O risco é mensurado por meio do desvio padrão dos resultados inesperados, ou "Sigma" $(\sigma)$, também chamado de volatilidade."

Através da análise dos resultados obtidos pelo desvio padrão, podemos concluir que quanto menor for o valor do desvio padrão, menor será a dispersão dos dados em relação à média, logo, menor será a volatilidade apresentada.

Segundo ASSAF NETO (2014) o conceito de risco pode ser dado pela dispersão do valor esperado do ativo em relação a sua média em um dado período, dispersão esta estudada através do desvio padrão. O autor cita em seu estudo que por meio da diversificação é possível esperar que ativos com risco possam ser combinados no contexto de uma carteira de forma que se apure um risco menor que aquele calculado para cada um de seus componentes.

Para isso, o autor classifica dois tipos fundamentais de risco, o Risco Sistemático e Risco Não Sistemático (Diversificável).

ASSAF NETO (2014) cita que o Risco Sistemático é aquele que não pode ser evitado 
por meio da diversificação do portfólio, pois tem origem nas flutuações que afetam o sistema de forma geral.

"Mesmo que as consequências das condições econômicas sejam perfeitamente entendidas, influencias não-econômicas podem mudar o curso da prosperidade geral, o nível do mercado ou o sucesso de uma ação em particular. A saúde do presidente, mudanças na tensão internacional, aumento ou redução no gasto militar, um verão extremamente seco [...] - tudo pode afetar o ganho de capital ou dividendos de uma ou mais ações.” (Markowitz, 1959, pg. 4)

Já o risco Não Sistemático, segundo ASSAF NETO (2014) pode ser reduzido por meio da diversificação, pois está relacionado mais diretamente com características do título e do mercado de negociação.

\subsection{MODELO DE MARKOWITZ}

Harry Markowitz publicou em 1952 no The Journal of Finance um artigo com o título "Seleção de Portfólio". Nele Markowitz inicia os estudos de carteiras de investimento e introduz a Teoria Moderna de Portfolios. Estes estudos apresentaram pela primeira vez um embasamento teórico matemático em relação à redução de risco resultante da diversificação (Marques et al, citação de COSTA; ASSUNÇÃO, 2005).

A teoria moderna de portfólio, propõe a composição de uma carteira de investimentos ótima através da diversificação dos ativos e uma relação positiva entre risco e retorno (MARQUES et al. 2013). Neste contexto, Markowitz (1952) foi pioneiro no conceito da diversificação, pois àquela época implementou o conceito de que não se deve investir em um único tipo de ativo, pois ao mesmo tempo em que se pode ter lucro considerável, a perda pode chegar a ser total, caso ocorra algum colapso neste investimento.

\subsubsection{Fronteira Eficiente}

A aplicação do modelo de Markowitz (1952), leva em conta o cálculo do retorno esperado e a variância entre os ativos, de acordo com o peso de cada ativo na carteira, conforme as fórmulas a seguir:

$$
\begin{aligned}
& E=\sum_{\substack{i=1 \\
n}}^{n} x_{i} \mu_{i} \\
& V=\sum_{i=1}^{n} \sum_{i=1}^{n} x_{i} x_{j} \sigma_{i j} \\
& \sum_{\substack{i=1 \\
x_{i}}}^{n} x_{i}=1
\end{aligned}
$$

Onde:

$E=$ Retorno Esperado da Carteira

$x_{i}=$ Peso do Ativo na Carteira

$V=$ Variância da Carteira

$$
\sigma_{i j}=\text { Covariância entre } x_{i} \text { e } x_{i}
$$

Pode-se determinar diversas carteiras de investimentos com uma determinada gama de 
ativos que mostrem o risco e o retorno de cada carteira. Os portfolios gerados são dados pelos pesos de proporção de cada ativo dentro carteira, e através do estudo de Teoria Moderna de Portfolio, encontra-se a fronteira eficiente, que se dá pela carteira com a melhor relação risco e retorno, conforme o Gráfico 1.

Este gráfico mostra uma aplicação prática da relação entre os ativos, medidos pela correlação. A carteira de ativos que possuem correlação igual a 1 são afetadas pelos mesmos fatores de mercado, por isso, pode ser visto uma maior exposição ao risco. Quanto mais a composição de carteiras tende à correlação igual a -1 , podemos perceber uma menor exposição ao risco, mostrando os efeitos da diversificação de portfólio estudada por Markowitz (1959). No mercado financeiro, não existem ativos com correlação igual a -1 (Perfeitamente Negativas).

Gráfico 1: Fronteira Eficiente de Markowitz

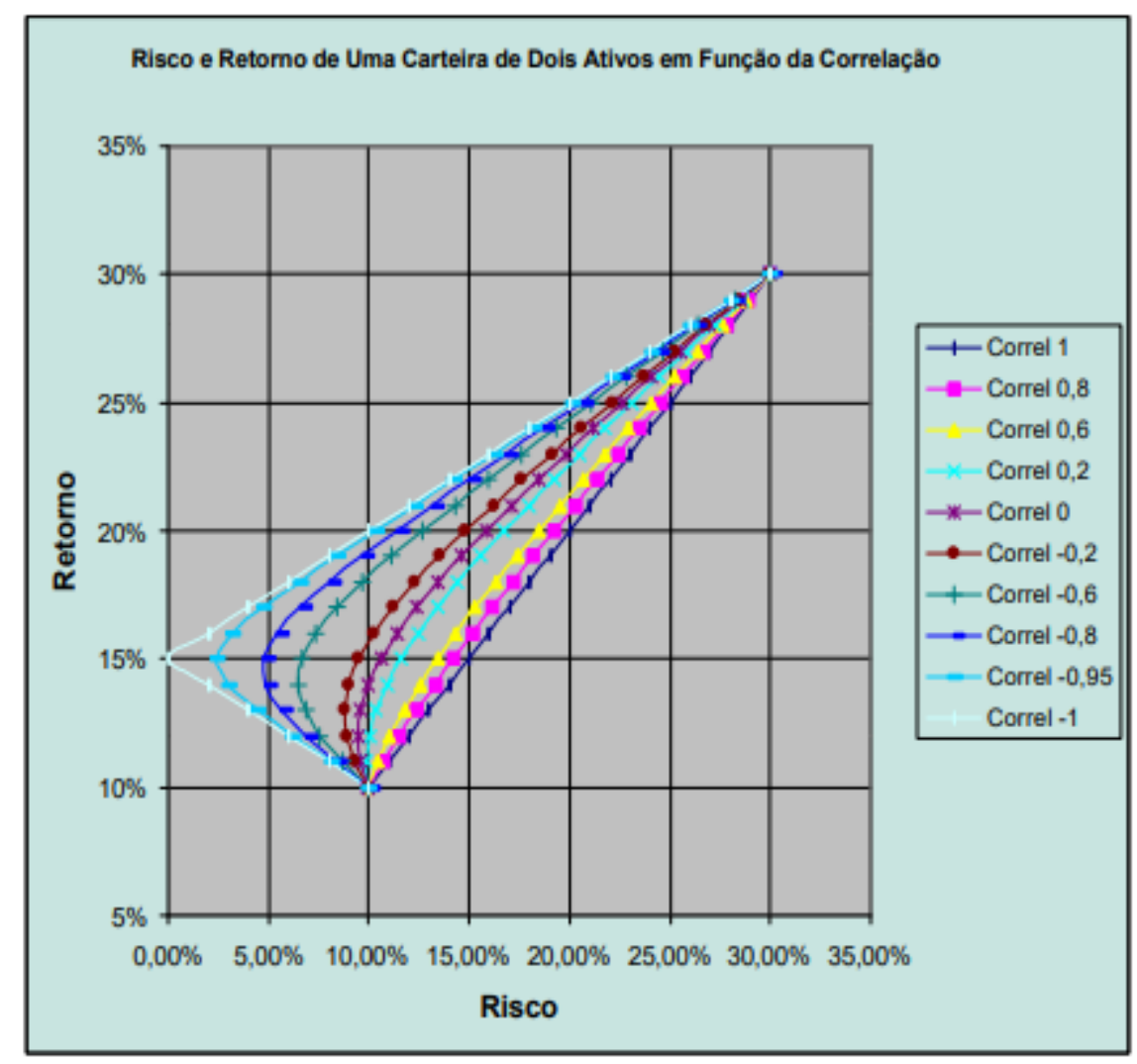

Fonte: Gonçalves Junior, Montevechi e Pamplona, 2002.

\section{5. ÍNDICE SHARPE}

O índice de performance de Sharpe, criado por William F. Sharpe em 1965, é definido como Índice de Recompensa pela Variabilidade "Reward to Variability Ratio e mede o retorno de uma carteira em relação ao risco da carteira.

Sharpe considerou a possibilidade de os investidores manterem parte de seus recursos aplicados em ativos com risco e parte aplicados em ativos livres de risco. O índice é calculado através da subtração entre retorno esperado do ativo (ou da carteira) e a risk free rate e a divisão pelo desvio padrão do mesmo, quanto maior for o resultado desta divisão melhor será relação entre o risco e o retorno. 


$$
I S=\frac{E(x)_{c}-R F R}{\sigma_{e}}
$$

Onde:

IS = Índice Sharpe

$E(x)_{c}=$ Retorno Esperado da Carteira

$R F R=$ Risk Free Rate

$\sigma_{\varepsilon}=$ Desvio Padrão da Carteira

\section{APLICAÇÃO DO MODELO DE MARKOWITZ}

Este trabalho está sendo realizado com base em revisão bibliográfica e aplicação empírica do modelo de Markowitz no mercado de Criptomoedas.

São utilizados os rendimentos mensais das cinco criptomoedas com maior volume de capitalização em dólar do período de julho de 2017 a junho de 2018, ranqueadas da seguinte forma:

Tabela 1. Criptomoedas e total Capitalizado de Jul/17 a Jun/18.

\begin{tabular}{|c|c|c|c|}
\hline$\#$ & Nome & Código & Capitalização \\
\hline 1 & Bitcoin & BTC & $\$ 147,91 \mathrm{~B}$ \\
\hline 2 & Ethereum & ETH & $\$ 27,34 \mathrm{~B}$ \\
\hline 3 & Ripple & XRP & $\$ 17,81 \mathrm{~B}$ \\
\hline 4 & Bitcoin Cash & BCH & $\$ 7,62 \mathrm{~B}$ \\
\hline 5 & Litecoin & LTC & $\$ 6,76 \mathrm{~B}$ \\
\hline
\end{tabular}

Fonte: Elaborada pelo autor através dos dados extraídos de https://br.investing.com/ em 30/05/2019.

Os dados foram extraídos da plataforma INVESTING.COM, site onde são fornecidos os dados históricos sobre os ativos de acordo com o período desejado em livre consulta. Os dados sobre retorno mensal das criptomoedas são apresentados na Tabela 2.

Tabela 2. Retorno Mensal das Moedas Virtuais.

\begin{tabular}{|c|c|c|c|c|c|}
\hline DATA & BITCOIN & ETHEREUM & RIPPLE & LITECOIN & BITCOIN CASH \\
\hline jul/17 & $16,23 \%$ & $-27,14 \%$ & $-33,17 \%$ & $7,04 \%$ & $0,00 \%$ \\
\hline ago/17 & $64,23 \%$ & $92,87 \%$ & $57,34 \%$ & $73,24 \%$ & $0,00 \%$ \\
\hline set/17 & $-7,91 \%$ & $-21,65 \%$ & $-22,91 \%$ & $-24,54 \%$ & $-26,27 \%$ \\
\hline out/17 & $47,94 \%$ & $0,08 \%$ & $-1,20 \%$ & $0,27 \%$ & $-0,51 \%$ \\
\hline nov/17 & $54,18 \%$ & $42,32 \%$ & $19,29 \%$ & $54,88 \%$ & $201,62 \%$ \\
\hline dez/17 & $39,25 \%$ & $70,47 \%$ & $740,41 \%$ & $164,71 \%$ & $79,73 \%$ \\
\hline jan/18 & $-25,88 \%$ & $51,75 \%$ & $-42,46 \%$ & $-27,86 \%$ & $-36,76 \%$ \\
\hline fev/18 & $0,67 \%$ & $-23,75 \%$ & $-22,15 \%$ & $23,27 \%$ & $-19,55 \%$ \\
\hline mar/18 & $-32,86 \%$ & $-53,79 \%$ & $-43,53 \%$ & $-42,41 \%$ & $-42,91 \%$ \\
\hline abr/18 & $33,25 \%$ & $70,09 \%$ & $65,98 \%$ & $27,49 \%$ & $96,62 \%$ \\
\hline mai/18 & $-18,85 \%$ & $-13,76 \%$ & $-26,50 \%$ & $-20,13 \%$ & $-26,00 \%$ \\
\hline jun/18 & $-14,71 \%$ & $-21,67 \%$ & $-23,92 \%$ & $-31,39 \%$ & $-24,88 \%$ \\
\hline
\end{tabular}

Fonte: Elaborada pelo autor através dos dados extraídos de https://br.investing.com/ 


\subsection{ESTATÍSTICAS DAS CRIPTOMOEDAS}

Neste estudo são utilizados o retorno médio e o desvio padrão de cada ativo para o período estudado dados na Tabela 3:

Tabela 3 - Retorno médio e desvio padrão das criptomoedas no período de jul/17 a jun/18.

\begin{tabular}{|c|c|c|}
\hline Ativo & $E[R]$ & $\sigma[R]$ \\
\hline Bitcoin & $12,96 \%$ & 0,3245 \\
\hline Ethereum & $13,82 \%$ & 0,4655 \\
\hline Ripple & $55,60 \%$ & 2,0941 \\
\hline Litecoin & $17,05 \%$ & 0,5622 \\
\hline Bitcoin Cash & $16,76 \%$ & 0,6984 \\
\hline
\end{tabular}

Fonte: Elaborada pelo autor através dos dados extraídos de https://br.investing.com/

A aplicação da teoria de Markowitz permite obter uma carteira ótima para uma taxa de retorno mínimo desejado, retorno este que o investidor realiza sem que seja necessário a exposição ao risco, esta taxa é chamada de Risk Free Rate, dada por um ativo que oferece uma remuneração sem que o investidor necessite se expor a qualquer tipo de risco.

O ativo livre de risco utilizado será a Taxa SELIC, definida pelo COPOM (Comitê de Políticas Monetárias), é a taxa média dos financiamentos diários, com lastro em títulos federais, taxa esta que influencia diretamente no rendimento dos títulos oferecidos pelo tesouro nacional, bancos privados e também pelo rendimento da poupança.

Este estudo foi realizado considerando a média das taxas mensais do período estudado. No início de 2017 o Banco Central implementou uma política monetária de redução contínua da taxa de juros, movimento este que se estabilizou em abril de 2018, fixando a taxa de juros em $6,50 \%$. Para efeito de comparação é efetuada a soma dos retornos mensais do período estudado, a fim de encontrar a taxa que reflita o retorno da Selic no período. Os dados históricos e a taxa acumulada no período serão mostrados na Tabela 4. 
Tabela 4. Taxa Selic.

\begin{tabular}{|c|c|}
\hline & Taxa \\
\hline jul/17 & $0,80 \%$ \\
\hline ago/17 & $0,80 \%$ \\
\hline set/17 & $0,64 \%$ \\
\hline out/17 & $0,64 \%$ \\
\hline nov/17 & $0,57 \%$ \\
\hline dez/17 & $0,54 \%$ \\
\hline jan/18 & $0,58 \%$ \\
\hline fev/18 & $0,47 \%$ \\
\hline mar/18 & $0,53 \%$ \\
\hline abr/18 & $0,52 \%$ \\
\hline mai/18 & $0,52 \%$ \\
\hline jun/18 & $0,52 \%$ \\
\hline Acum. & $7,37 \%$ \\
\hline
\end{tabular}

Fonte: CETIP. Extraído de: http://estatisticas.cetip.com.br/astec/series v05/paginas/lum web v04 1003 consulta.asp

Logo, a Risk Free Rate utilizada neste trabalho será dada pelo acumulado dos rendimentos mensais da taxa Selic no período, ou seja, Risk Free Rate igual a 7, 37\%.

A matriz de covariância é elaborada a partir dos dados históricos do retorno dos ativos, ela pode ser construída pela aplicação direta da ferramenta do MS Excel, realizada neste trabalho. A Matriz de Covariância encontrada será mostrada na Tabela 5:

Tabela 5. Matriz de Covariância.

\begin{tabular}{|l|c|c|c|c|c|}
\hline & BITCOIN & ETHEREUM & RIPPLE & LITECOIN & BITCOIN CASH \\
\hline BITCOIN & 0,105308056 & 0,099417977 & 0,252386604 & 0,130813813 & 0,15039193 \\
\hline ETHEREUM & 0,099417977 & 0,216690136 & 0,472273298 & 0,178463877 & 0,170668536 \\
\hline RIPPLE & 0,252386604 & 0,472273298 & 4,385129338 & 1,014812199 & 0,53220983 \\
\hline LITECOIN & 0,130813813 & 0,178463877 & 1,014812199 & 0,316102207 & 0,231359545 \\
\hline BITCOIN CASH & 0,15039193 & 0,170668536 & 0,53220983 & 0,231359545 & 0,487777597 \\
\hline \multicolumn{7}{|c|}{ Fonte: Elaborada pelo autor }
\end{tabular}

\subsection{IMPLEMENTAÇÃO DO MODELO DE MARKOWITZ E OS RESULTADOS OBTIDOS}

Outro ponto importante estudado no trabalho de Markowitz é o peso das ações na carteira. Por isso como critério inicial definimos pesos iguais dos ativos na carteira, para que seja encontrado a partir do modelo de otimização o peso ideal de cada ativo na carteira maximizando a relação risco-retorno da mesma.

Para esta carteira é feito o cálculo retorno esperado, do desvio padrão e índice Sharpe, com a finalidade de encontrar a relação entre o risco e retorno da carteira inicialmente estudada. Cálculo este mostrado na Tabela 7, onde temos os valores de retorno esperado e desvio padrão da carteira considerando o mesmo peso de $20 \%$ para cada ativo. 
Tabela 7. Retorno esperado, desvio padrão e índice Sharpe da carteira no período de Jul/17 a Jun/18.

\begin{tabular}{|c|c|}
\hline Medida & Retorno \\
\hline $\mathrm{E}[\mathrm{R}]$ & $23,24 \%$ \\
\hline Desvio Padrão $(\sigma)$ & $69,21 \%$ \\
\hline Índice Sharpe & 0,2292 \\
\hline
\end{tabular}

Fonte: Elaborada pelo autor

Neste cenário, obteve-se o retorno da carteira de 23,24\% e o Desvio Padrão de 69,21\%, o que nos apresenta um grau de volatilidade elevado deste conjunto de ativos. O índice Sharpe calcula a relação entre estas duas medidas e quanto maior for o seu valor, melhor será a relação entre o risco retorno.

Com a finalidade de otimizar a carteira de ativos, utiliza-se a ferramenta Solver do MS Excel para desenvolver o estudo como um problema de programação não linear, sendo possível definir a função objetivo e as restrições do problema, para que seja encontrada solução que otimize os resultados esperados na função objetivo.

Para definir a função objetivo, retorna-se aos conceitos estudados por Markowitz (1952 e 1959) que busca minimizar o risco a fim de encontrar a melhor relação risco e retorno. Portanto, é definido como função objetivo do problema minimizar a variância da carteira. Conforme a fórmula de cálculo da variância temos, portanto, uma função objetivo quadrática sujeito a restrições lineares, dadas pelas seguintes condições:

O somatório dos pesos dos ativos deve ser igual a $1(100 \%)$.

O retorno esperado da carteira ser igual ao retorno definido.

O modelo de otimização quadrática a ser aplicado é:

$$
\operatorname{Min} \mathrm{z}=\sum_{\substack{\mathrm{i}=1 \\ \mathrm{~S} . \mathrm{a}}}^{n} \sum_{j=1}^{n} x_{i} x_{j} \sigma_{i j}
$$

$$
\sum_{i=1}^{n} x_{i}=1
$$$$
\sum_{i=1}^{n} E[R]_{i} \cdot x_{i} \geq r
$$

$x_{i} \geq 0$

Onde:

$r=$ Retorno Mínimo Estipulado pelo Investidor

Com os padrões definidos, utilizamos o Solver para calcular a carteira otimizada através do modelo de Markowitz para o retorno esperado encontrado na carteira inicial.

Para este modelo, tivemos o seguinte resultado: 
Tabela 8. Composição da Carteira Ótima. $r=23,24 \%$.

\begin{tabular}{|l|r|l|r|}
\hline \multicolumn{1}{|c|}{ Ativo } & Peso & \multicolumn{1}{c|}{ Medidas } & Resultado \\
\hline Bitcoin & $76 \%$ & SELIC & $7,37 \%$ \\
\hline Ethereum & $0 \%$ & E[R] & $23,24 \%$ \\
\hline Ripple & $24 \%$ & Var & $40,80 \%$ \\
\hline Litecoin & $0 \%$ & Desvp & $63,88 \%$ \\
\hline Bitcoin Cash & $0 \%$ & IS & 0,2485 \\
\hline
\end{tabular}

Fonte: Elaborada pelo autor

Neste caso, a carteira seria composta por $76 \%$ de Bitcoin e $24 \%$ de Ripple, reduzindo o risco em aproximadamente 5,39\% e obtendo um leve aumento do índice Sharpe.

$\mathrm{Na}$ sessão seguinte, serão obtidos seis modelos diferentes da ferramenta, cada um fixando um retorno para os investimentos, para que possa ser analisado o risco correspondente da carteira. Sendo assim, são utilizados os seguintes retornos:

Tabela 9. Retornos escolhidos para os modelos implementados.

\begin{tabular}{|c|c|}
\hline$\#$ & Retorno Definido \\
\hline 1 & $15,00 \%$ \\
\hline 2 & $20,00 \%$ \\
\hline 3 & $25,00 \%$ \\
\hline 4 & $30,00 \%$ \\
\hline 5 & $35,00 \%$ \\
\hline 6 & $40,00 \%$ \\
\hline
\end{tabular}

Fonte: Elaborada pelo autor

\subsubsection{Primeira Hipótese. $r=15,00 \%$}

Utilizando $r=15,00 \%$, ao selecionar a opção "RESOLVER" no Solver para o primeiro retorno temos o seguinte panorama:

Tabela 10. Composição da Carteira ótima para $r=15 \%$.

\begin{tabular}{|c|c|c|c|}
\hline Ativo & Peso & Medidas & Resultado \\
\hline Bitcoin & $94 \%$ & SELIC & $7,37 \%$ \\
\hline Ethereum & $2 \%$ & $E[R]$ & $15,00 \%$ \\
\hline Ripple & $5 \%$ & Var & $12,85 \%$ \\
\hline Litecoin & $0 \%$ & Desvp & $35,84 \%$ \\
\hline Bitcoin Cash & $0 \%$ & IS & 0,2129 \\
\hline
\end{tabular}

Fonte: Elaborada pelo autor

Após a resolução do Solver, observa-se que a minimização da função objetivo foi realizada e pela solução encontrada, observa-se que $94 \%$ do peso da carteira seria aplicado em Bitcoin, 2\% em Ethereum e 5\% em Ripple

Para este cenário, obteve-se um retorno da carteira de $15,00 \%$, desvio padrão de $35,84 \%$ e um índice Sharpe de 0,2129.

\subsubsection{Segunda Hipótese. $r=20,00 \%$}


Utilizando $r=20,00 \%$, ao selecionar a opção "RESOLVER" no solver para o segundo retorno temos o seguinte panorama:

Tabela 11. Composição da Carteira ótima para $r=20 \%$.

\begin{tabular}{|l|r|l|r|}
\hline \multicolumn{1}{|c|}{ Ativo } & Peso & \multicolumn{1}{c|}{ Medidas } & Resultado \\
\hline Bitcoin & $83 \%$ & SELIC & $7,37 \%$ \\
\hline Ethereum & $0 \%$ & E[R] & $20,00 \%$ \\
\hline Ripple & $17 \%$ & Var & $26,26 \%$ \\
\hline Litecoin & $0 \%$ & Desvp & $51,24 \%$ \\
\hline Bitcoin Cash & $0 \%$ & IS & 0,2465 \\
\hline
\end{tabular}

Fonte: Elaborada pelo autor

Após a resolução do Solver, observa-se que a minimização da função objetivo foi realizada, observa-se que $83 \%$ do peso da carteira seria aplicado em Bitcoin e $17 \%$ em Ripple.

Para este cenário, obteve-se um retorno da carteira de $20,00 \%$, desvio padrão de $51,24 \%$ e um índice Sharpe de 0,2465.

\subsubsection{Terceira Hipótese. $r=25,00 \%$}

Utilizando $\mathrm{r}=25,00 \%$, ao selecionar a opção "RESOLVER" no solver para o terceiro retorno temos o seguinte panorama:

Tabela 12. Composição da Carteira ótima para $\mathrm{r}=25 \%$.

\begin{tabular}{|l|r|l|r|}
\hline \multicolumn{1}{|c|}{ Ativo } & Peso & \multicolumn{1}{c|}{ Medidas } & Resultado \\
\hline Bitcoin & $71 \%$ & SELIC & $7,37 \%$ \\
\hline Ethereum & $0 \%$ & E[R] & $25,00 \%$ \\
\hline Ripple & $28 \%$ & Var & $50,63 \%$ \\
\hline Litecoin & $0 \%$ & Desvp & $71,16 \%$ \\
\hline Bitcoin Cash & $1 \%$ & IS & 0,2478 \\
\hline \multicolumn{2}{|c|}{ Fonte: Elaborada pelo autor } \\
\hline
\end{tabular}

Após a resolução do Solver, observa-se que a minimização da função objetivo foi realizada e pela solução encontrada, observa-se que $71 \%$ do peso da carteira seria aplicado em Bitcoin, 28\% em Ripple e 1\% em Bitcoin Cash

Para este cenário, obteve-se um retorno da carteira de $40,00 \%$, desvio padrão de $71,16 \%$ e um índice Sharpe de 0,2478.

\subsubsection{Quarta Hipótese. $r=30,00 \%$.}

Utilizando $\mathrm{r}=30,00 \%$, ao selecionar a opção "RESOLVER" no solver para o quarto retorno temos o seguinte panorama: 
Tabela 13. Composição da Carteira ótima para $\mathrm{r}=30 \%$.

\begin{tabular}{|l|r|l|r|}
\hline \multicolumn{1}{|c|}{ Ativo } & Peso & Medidas & Resultado \\
\hline Bitcoin & $55 \%$ & SELIC & $7,37 \%$ \\
\hline Ethereum & $0 \%$ & $E[R]$ & $30,00 \%$ \\
\hline Ripple & $39 \%$ & Var & $85,88 \%$ \\
\hline Litecoin & $0 \%$ & Desvp & $92,67 \%$ \\
\hline Bitcoin Cash & $6 \%$ & IS & 0,2442 \\
\hline \multicolumn{3}{|c}{ Fonte: Elaborada pelo autor }
\end{tabular}

Após a resolução do Solver, observa-se que a minimização da função objetivo foi realizada e pela solução encontrada, observa-se que $55 \%$ do peso da carteira seria aplicado em Bitcoin, 39\% em Ripple e 6\% em Bitcoin Cash

Para este cenário, obteve-se um retorno da carteira de 30,00\%, desvio padrão de 92,67\% e um índice Sharpe de 0,2442.

\subsubsection{Quinta Hipótese. $r=35,00 \%$.}

Utilizando $r=35,00 \%$, ao selecionar a opção "RESOLVER" no solver para o quarto retorno temos o seguinte panorama:

Tabela 14. Composição da Carteira ótima para $r=35 \%$.

\begin{tabular}{|c|c|}
\hline Ativo & Peso \\
\hline Bitcoin & $48 \%$ \\
\hline Ethereum & $0 \%$ \\
\hline Ripple & $52 \%$ \\
\hline Litecoin & $0 \%$ \\
\hline $\begin{array}{c}\text { Bitcoin } \\
\text { Cash }\end{array}$ \\
\hline
\end{tabular}

\begin{tabular}{|l|r|l|r|}
\hline \multicolumn{1}{|c|}{ Ativo } & Peso & \multicolumn{1}{|c|}{ Medidas } & Resultado \\
\cline { 2 - 3 } Bitcoin & $39 \%$ & SELIC & $7,37 \%$ \\
\hline Ethereum & $0 \%$ & E[R] & $35,00 \%$ \\
\hline Ripple & $51 \%$ & Var & $131,96 \%$ \\
\hline Litecoin & $0 \%$ & Desvp & $114,87 \%$ \\
\hline Bitcoin Cash & $11 \%$ & IS & 0,2405 \\
\hline \multicolumn{3}{|c|}{ Fonte: Elaborada pelo autor } \\
\hline \multicolumn{3}{|c|}{}
\end{tabular}

Após a resolução do Solver, observa-se que a minimização da função objetivo foi realizada e pela solução encontrada, observa-se que 39\% do peso da carteira seria aplicado em Bitcoin, 51\% em Ripple e 11\% em Bitcoin Cash.

Para este cenário, obteve-se um retorno da carteira de $35,00 \%$, desvio padrão de $114,87 \%$ e um índice Sharpe de 0,2405.

\subsubsection{Sexta Hipótese. $r=40,00 \%$.}

Tabela 15. Composição da Carteira ótima para $r=40 \%$. 


\begin{tabular}{|l|r|l|r|}
\hline \multicolumn{1}{|c|}{ Ativo } & Peso & \multicolumn{1}{c|}{ Medidas } & Resultado \\
\hline Bitcoin & $22 \%$ & SELIC & $7,37 \%$ \\
\hline Ethereum & $0 \%$ & E[R] & $40,00 \%$ \\
\hline Ripple & $62 \%$ & Var & $188,86 \%$ \\
\hline Litecoin & $0 \%$ & Desvp & $137,43 \%$ \\
\hline Bitcoin Cash & $16 \%$ & IS & 0,2374 \\
\hline \multicolumn{3}{|c|}{ Fonte: Elaborada pelo autor }
\end{tabular}

Após a resolução do Solver, observa-se que a minimização da função objetivo foi realizada e pela solução encontrada, observa-se que $22 \%$ do peso da carteira seria aplicado em Bitcoin, 62\% em Ripple e 16\% em Bitcoin Cash.

Para este cenário, obteve-se um retorno da carteira de $40,00 \%$, desvio padrão de $137,43 \%$ e um índice Sharpe de 0,2374.

\section{ANÁLISE DOS RESULTADOS OBTIDOS}

A partir dos resultados obtidos, observa-se o comportamento do risco em relação ao retorno definido. Durante todo o período, desde o início das negociações das criptomoedas, uma característica sempre presente neste mercado é a volatilidade, os dados históricos mostram uma grande variação do valor em um curto espaço de tempo. O estudo de Markowitz nos confirma o grande risco inerente a este tipo de investimento.

Analisando pelo índice Sharpe da carteira, a medida em que o retorno esperado aumenta o resultado encontrado para o índice também aumenta, o que aparenta melhora na relação risco e retorno, mas ainda assim, na última carteira estudada encontramos um desvio padrão de $137,43 \%$, considerado muito acima do comum encontrado no mercado financeiro. Por mais que haja uma melhora na relação, dada pelo índice Sharpe, o valor encontrado ainda está muito aquém para aceitação ao risco.

As primeiras carteiras, que possuem um retorno de $15 \%$ e $20 \%$ respectivamente, mostram um risco aceitável para o investidor, porém, com a composição de carteiras com o retorno acima de $25 \%$, podemos analisar carteiras que assumem uma composição de risco muito elevado, sendo de caráter atrativo apenas para investidores arrojados, que buscam retornos significativos se expondo a altos índices de risco. Na última carteira, observa-se uma composição de $22 \%$ de Bitcoin, $62 \%$ de Ripple e $18 \%$ em Bitcoin Cash para um retorno de $40 \%$, o que dentro das expectativas de mercado é um retorno considerável para o período estudado, considerando que o IBOVESPA (Índice da Bolsa de Valores de São Paulo) para o mesmo período teve um retorno de $9,40 \%$.

\section{CONCLUSÃO}

A aplicação do modelo estudado nos mostra que a formação de carteira de investimentos de Criptomoedas traz consigo uma medida de risco constantemente alta. Todas as formações de portfólio mostram um bom retorno da carteira, porém deve ser adequado à um perfil de investidor que tenha considerável apetite ao risco.

Por mais que o modelo de Markowitz nos ofereça diversas carteiras que relacionam o risco ao retorno, em todas as tentativas, a volatilidade das criptomoedas se mostrou muito elevada para os padrões trabalhados no mercado financeiro.

O autor ULRICH (2014) afirmou que a volatilidade diminuiria conforme o aumento 
de investidores no mercado de criptomoedas. Podemos observar com este estudo, que o mercado ganhou muitos adeptos até o ano de 2018, mas que ainda não resultou na redução da volatilidade deste tipo de investimento.

Contudo, o estudo se mostrou viável e pelo fato deste mercado ganhar muitos investidores nos últimos anos, em sua maioria com grande apetite ao risco, neste contexto o método se mostrou eficaz em expor ao investidor a relação de risco e retorno dos ativos, indicando uma carteira ótima para realizar os investimentos.

\section{REFERÊNCIAS BIBLIOGRÁFICAS}

ALMEIDA, N.; SILVA, R.; RIBEIRO, K. Aplicação do Modelo de Markowitz na Seleção de Carteiras Eficientes: Uma Análise de Cenários no Mercado de Capitais Brasileiro, XIII Semead. Minas Gerais. 2010.

ANDERSON, D. R., SWEENEY, D. J \& WILLIANS, T. A. Estatística Aplicada à Administração e Economia. 2a Edição. São Paulo. 2002.

ASSAF NETO. Mercado Financeiro. São Paulo: Atlas, 12ª edição. 2014.

CASAROTTO FILHO, N.; KOPITTKE, B. H. Análise de Investimentos: Matemática Financeira, Engenharia Econômica, Tomada de Decisão, Estratégia Empresarial. São Paulo: Atlas, 2010. 411 p.

COLIN, E. Pesquisa Operacional: 170 aplicações em estratégia, finanças, logística, produção, marketing e vendas. Rio de Janeiro: LTC, 2011.

COSTA, O.; ASSUNÇÃO, H. Análise de Risco e retorno em investimentos financeiros. Barueri: Manole, 2005.

CROSBY, M., PATTANAYAK, P., VERMA, S. and KALYANARAMAN, V. Blockchain technology: Beyond bitcoin. Applied Innovation, 2:6-10. 2016.

ELTON, E. J.; GRUBER, M. J.; BROWN, S. J.; GOETZMANN, W. N. Moderna Teoria de Carteiras e Análise de Investimentos. São Paulo: Atlas, 2004.

FIGUEIREDO, A. C. et al. A utilização da teoria de carteiras de Markowitz e do modelo de índice único de Sharpe no mercado de ações brasileiro em 1999. São Paulo: Resenha BM\&F - nº 141, 2000.

FONSECA, C. G. Aplicação do Modelo de Markowitz na Seleção de Carteiras Eficientes: Uma análise da Relação entre Risco e Retorno. Trabalho de Conclusão do Curso (Especialização). MBA em Finanças e Gestão de Risco. Universidade Federal do Rio de Janeiro, 2011.

GONÇALVES JUNIOR, C.; MONTEVECHI, J. A. B.; PAMPLONA, E. O. Seleção de carteiras através do modelo de Markowitz para pequenos investidores (com o uso de planilhas eletrônicas). Bauru, SP, IX Simpep outubro 2002.

JORION, P. Value at risk: a nova fonte de referência para o controle de risco de mercado. São Paulo: BM\&F, 1998.

LIMA, M. G. B., GUIMARÃES JÚNIOR, D. S., TAVORA JÚNIOR, J. L. Utilização de Pesquisa Operacional para determinação da Carteira de Variância Mínima no Mercado Acionário. In Anais do XXX Encontro Nacional de Engenharia de Produção, São Carlos, SP, BR, 2010. 
MARKOWITZ, H. M. Portfolio Selection. Journal of Finance, v. 7, 77-91, 1952.

MARKOWITZ, H. M. Portfolio Selection. Efficient Diversification of Investiment, Londres, 1959.

MARQUES, S.; SILVA, W. V.; DEL CORSO, J. M.; DALAZEN, L. L. Comparação de Desempenhos de Carteiras Otimizadas pelo Modelo de Markowitz e a Carteira de Ações do IBOVESPA. Revista Evidenciação Contábil \& Finanças. João Pessoa, v.1, n. 1, p. 20-37, jan./jun. 2013.

MASCARENHAS, J. Z. G.; VIEIRA, A. B.; ZIVIANI, A. Análise da Rede de Transações do Ethereum. In:, 1. , 2018, 1/2018. Anais do I Workshop em Blockchain: Teoria, Tecnologias e Aplicações (WBlockchain - SBRC 2018). Porto Alegre: Sociedade Brasileira de Computação, maio 2018.

PEREIRA, L. B. T., HENRIQUE, D. C. Otimização de Investimentos pelo Modelo de Markowitz via desenvolvimento de uma ferramenta em Excel. Iberoamerican Journal of Industrial Engineering, Florianópolis, SC, Brasil, v. 8, n.16, p.167-195, 2016.

SOUZA, L. C., MASSARDI, W. O., PIRES, V. A V., CIRIBELI, J. P. Otimização de Carteira de Investimentos: Um Estudo com Ativos do IBOVESPA. Revista de Gestão, Finanças e Contabilidade, UNEB, Salvador, v. 7, n. 3, p. 201-213, set./dez., 2017.

TAHA, A. H, Pesquisa Operacional, $8^{\mathrm{a}}$ EDIÇÃO. 2008

ULRICH, F. Bitcoin: A Moeda na Era Digital. Instituto Ludwig von Mises Brasil. São Paulo. 2014. 